\title{
Research on Displacement Calculation of Dynamometer Card Based on Kalman Filter and Discrete Numerical Integration
}

\author{
Nan Yana , Jia Shu Dai \\ School of Computer and Information, Anhui Polytechnic University, 241000 Wuhu, China
}

\begin{abstract}
In order to calculate the dynamometer card of oil well using acceleration sensor, the algorithm which combined by Kalman filter and discrete numerical integration is proposed. It can be applied to calculate the displacement and precipitation displacement period of oil well dynamometer card. The Kalman filter not only filters out the noise of the acceleration signal, but also maintains the original shape feature. The accurate precipitation of the displacement period ensures the correctness of displacement. The discrete numerical integration algorithm can make the relative error of displacement measurement less than $1 \%$, which meets the requirement for dynamometer card accuracy. It is suitable for different types of oil wells.
\end{abstract}

\section{Introduction}

The dynamometer card is a graph that reflects the change rules of polished rod load of pumping unit with its displacement. The real-time dynamic of oil well and the rationality of pumping unit device can be known through the analysis of dynamometer card. The dynamometer card is one of the important basis for the fault diagnosis of the pumping unit, and the displacement is the horizontal ordinate of the graph. The products for measuring dynamometer cards by acceleration sensors have been developed at abroad, but few of them are still available in china. At present, the modes of measuring the displacement and stroke which used in oil field are mainly wired and wireless. The wired measurement mode has high precision and good reproducibility. However, there are some shortcomings, such as heavy equipment, high breakage rate, serious local wear and so on, these resulting in a lot of inconvenience to field operations ${ }^{[1]}$. The wireless measurement mode is mainly measured by the inclination sensor and the acceleration sensor. The inclination and the acceleration sensor measure the displacement with the advantages of legerity and convenient, and are not interfered by the external environment. However, due to excessive vibration and resistance during the movement process of pumping unit, these caused the collected digital signals have a lot of noise and singular points (too large or too small signal). At present, the smoothing filter is used to calculate the displacement of acceleration signal in oil industry. This method is difficult to filter out the noise signal and remove the signal singular points, the calculated displacement measurement accuracy is low, the repetition rate is poor and the error is large ${ }^{[2]}$. How to obtain an accurate and small error displacement is the key to solve the problem of dynamometer card. Therefore, the algorithm which combined by Kalman filtering and discrete numerical integration is proposed to calculate the displacement and the precipitation displacement period of oil well dynamometer card, as follows.

\section{Filtering Processing}

The filtering algorithm is used to de-noise the original acceleration data so as to make the acceleration signal as smooth as possible. The vibration of pumping unit and resistance of downhole will cause the acceleration signal a lot of noise in the acceleration acquisition process. Thus, the displacement after second order integral will produce a great error. Therefore, filtering is essential before integration. The incoming interface of the acceleration signal will plus $0.1 \mathrm{uF}$ filter capacitor for hardware filtering. The Kalman filter is the first time applied to the displacement calculation of the dynamometer card after comparing the common 7-point smoothing filter and Kalman filter.

\subsection{The principle of 7-point moving average filtering}

The measurement data storage in the 7- point moving average filtering is the queue. The first seven points of the queue (length $\mathrm{N}$ ) to be processed is divided into a group, the results after processing is putted to the end of the queue while the header of the original queue is discarded. Then the latest $\mathrm{N}$ data remains in the queue. When the average is calculated, the arithmetic average of the seven data in the queue produces a new arithmetic mean. The new arithmetic averages can be obtained after

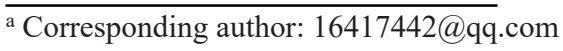


each time processing until the end of the queue. The three times 7-point moving average filtering is carried out on the RAM which stored 400 acceleration signals. The formula is as follows:

$$
X_{k}=\left(X_{k-3}+X_{k-2}+X_{k-1}+X_{k}+X_{k+1}+X_{k+2}+X_{k+3}\right)(3 \leq \mathrm{k} \leq \mathrm{N}-3)
$$

Where, $X_{k}$ is the k-th acceleration data, $\mathrm{N}$ is the number of data which collected in the entire process.

\subsection{The principle of Kalman filter and its modeling realization}

Kalman filter has been applied to robot control, sensor data fusion, navigation and other fields. It has also been widely used in image processing, and has the advantages of fast speed and high computational precision. Kalman filter is an iterative algorithm, while the minimum mean square error estimation is the best criterion ${ }^{[3]}$. The basic idea of Kalman filter is to use the signal and noise state space as the model under the best estimation criterion. The estimated values are obtained by update the state variable estimation using the last estimated value and current measured value. The discrete process systems and the measurement equations of the data to be processed are established to satisfy the best criteria. The Kalman filter modeling method is as follows.

Step 1 The discrete control process system is introduced which can be represented by a linear stochastic differential equation:

$$
X(k)=A X(k-1)+B U(k)+W(k)
$$

The measured values of the system

$$
Z(k)=H X(k)+V(k)
$$

In the formula (2) and (3), X (k) is the system state at time $\mathrm{k}, \mathrm{U}(\mathrm{k})$ is the control variable of the system at time $\mathrm{k}, \mathrm{A}$ and $\mathrm{B}$ are the system parameters. $\mathrm{Z}(\mathrm{K})$ is the measured value at time $\mathrm{k}, \mathrm{H}$ is the measurement system parameter. $\mathrm{W}(\mathrm{k})$ is the process noise, $\mathrm{V}(\mathrm{k})$ is the measurement noise, assuming these are Gaussian white noises, their covariance is $\mathrm{Q}, \mathrm{R}$ (here we assume that they do not change with the state of the system).

Step 2 Predict the current state. The Kalman filter is the most suitable algorithm for the Gaussian white noise system. The optimized output estimates are according to the covariance of the system. Set $\mathrm{k}$ is the current system state, the current state estimate value can be predicted by the system's previous state, the current estimate state is:

$$
X(k \mid k-1)=A X(k-1)+B U(k)
$$

In the formula (4), $\mathrm{X}(\mathrm{k} \mid \mathrm{k}-1)$ is the previous state prediction result. $\mathrm{U}(\mathrm{k})$ is the current state control variable, when there is no control variable, it is 0 . The system results are updated here, but the covariance of $\mathrm{X}$ ( $\mathrm{k} \mid \mathrm{k}-1)$ is not updated. $\mathrm{P}$ is the covariance; the current error is predicted by:

$$
\mathrm{P}(\mathrm{k} \mid \mathrm{k}-1)=\mathrm{AP}(\mathrm{k}-1) \mathrm{A}^{\mathrm{T}}+\mathrm{Q}
$$

In the formula (5), $\mathrm{P}(\mathrm{k} \mid \mathrm{k}-1)$ is the covariance of $\mathrm{X}$ $(\mathrm{k} \mid \mathrm{k}-1), \mathrm{P}(\mathrm{k}-1)$ is the covariance of $\mathrm{X}(\mathrm{k}-1), \mathrm{A}^{\mathrm{T}}$ is the transposed matrix of $\mathrm{A}$, the covariance of system process is $\mathrm{Q}$.

Step 3 Get the optimal estimate value $X(\mathrm{k}) . \mathrm{X}(\mathrm{k})$ is the current optimal estimate value (current state $\mathrm{k}$ ), the present state prediction results in step 2 can be obtained by combine the measured values and the previous predicted values;

The current optimal estimate value is:

$X(k)=X(k \mid k-1)+K g(k) *(Z(k)-H \quad X(k \mid k-1))(6)$

$\mathrm{Kg}$ is the Kalman gain,

$$
\mathrm{Kg}(\mathrm{k})=\frac{\mathrm{P}(\mathrm{k} \mid \mathrm{k}-1) \mathrm{H}^{\mathrm{T}}}{\left(\mathrm{H} \mathrm{P}(\mathrm{k} \mid \mathrm{k}-1) \mathrm{H}^{\mathrm{T}}+\mathrm{R}\right)}
$$

Here, $\mathrm{X}(\mathrm{k})$ is the optimal estimate value in the state $\mathrm{k}, \mathrm{P}(\mathrm{k})$ is updated (the covariance of $\mathrm{X}(\mathrm{k})$ ) so that the Kalman filter can be run continuously before the end of the system:

$$
\mathrm{P}(\mathrm{k})=((\mathrm{I}-\mathrm{Kg}(\mathrm{k}) \mathrm{H}) \mathrm{P}(\mathrm{k} \mid \mathrm{k}-1)
$$

The matrix I in formula (8) is 1(Single measurement of single model, $\mathrm{I}=1)$. When the system is updated to state $\mathrm{k}+1, \mathrm{P}(\mathrm{k})=\mathrm{P}(\mathrm{k}-1)$ (formula (5)). Keep running in an autoregressive manner.

The basic model of Kalman filter can be realized through the above three steps. The formulas (4), (5), (6), (7) and (8) are the five basic formulas of the Kalman filter, it is easy to code implement with these five formulas ${ }^{[4]}$. From the above, the current optimal estimate value $X(k)$ and the error value $P(k)$ can be obtained through the initial state $X_{0}$ and the initial error $P_{0}$, and the current observation value $Z(k)$. Figure 1 is the original acceleration data which collect from acceleration sensor, Figure 2 and Figure 3 are the effect comparison of Kalman filter and three times 7-point moving filter.

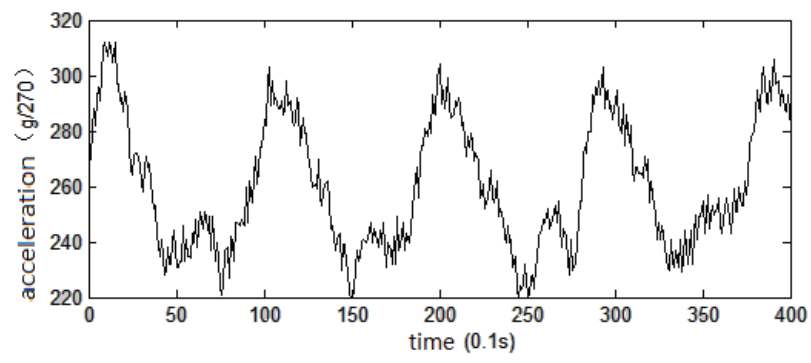

Figure 1. The original data of acceleration. 


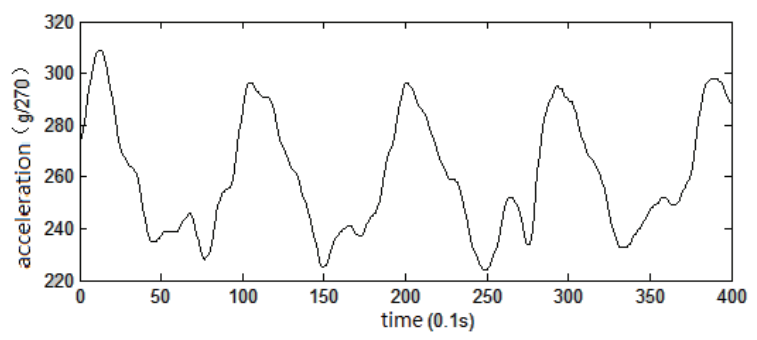

Figure 2. The data after Kalman filter.

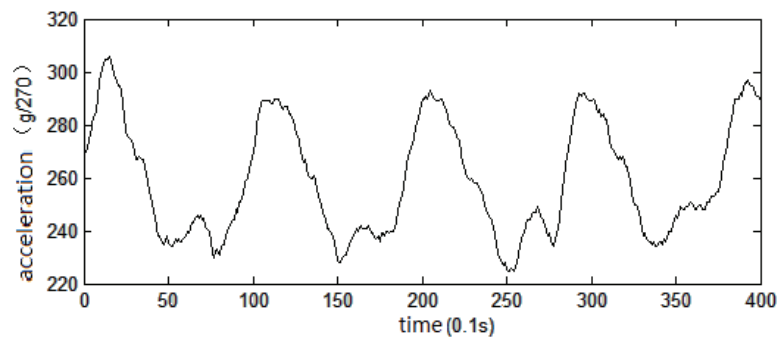

Figure 3. The data after 7-point moving filter.

By comparing Figure 2 and Figure 3, the Kalman filter described in Figure 2 is better to reflects realistic acceleration signal, the stroke measurement has a good repeatability in the same oil well. It can eliminate the singular points in the acceleration noise. The most outstanding advantage is that fast real-time processing and saving memory capacity.

\section{The displacement period is precipitated by the acceleration after filtering}

According to the periodic characteristics of sucker rod movement, the acceleration of 400 points are collected at a time, the acquisition interval is controlled at $100 \mathrm{~ms}$. In order to obtain at least one period of acceleration raw data of pumping unit at different speeds, once acquisition per 40s. Since the pumping rod of pumping unit makes periodic reciprocating motion, assuming that the movement period is $\mathrm{T}$, the movement regulation satisfies the following three formulas:

$$
\begin{gathered}
\mathrm{a}(0)=\mathrm{a}(\mathrm{T}) \\
\mathrm{v}(0)=\mathrm{v}\left(\frac{\mathrm{T}}{2}\right)=\mathrm{v}(\mathrm{T}) \\
\mathrm{d}(0)=\mathrm{d}(\mathrm{T})
\end{gathered}
$$

Among them, $\mathrm{a}(\mathrm{t})$ is the acceleration of sucker rod, $\mathrm{V}$ (t) is the speed of sucker rod, $d(t)$ is the relative displacement of the sucker rod.

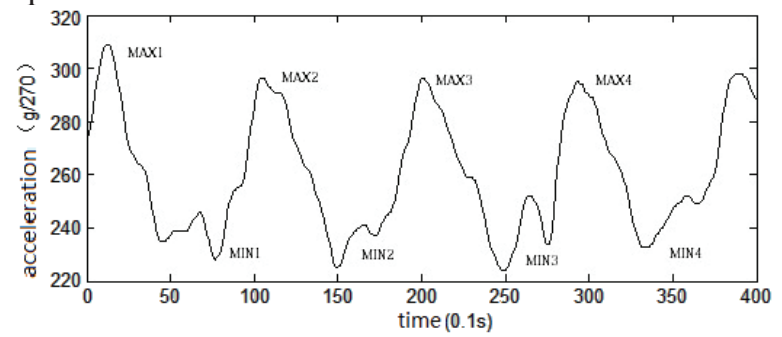

Figure 4. The period analysis chart.
In the process of pumping up and down, the speed at the maximum and minimum acceleration is 0 . As can be seen from Figure 4, the MAX set of acceleration is the highest point at the displacement of the pumping unit which is the zero point of relative displacement. Among them, MIN is the maximum point of relative displacement, and the two adjacent local maximum or minimum point is a displacement period. The algorithm for calculating the period is divided into the following four steps to ensure the correctness of the period.

Step 1 Look for the maximum and minimum values of the previous $2 / 3$ periods (this step ensures that the complete period can be found in the case of very little acceleration)

Step 2 Compare the subscripts of the maximum and minimum, if the subscript of the maximum value is close to 0 , go to (step 3), otherwise, go to (step 4).

Step 3 The begin point of the maximum acceleration subscript is recorded. As the MAX1 in figure, look backward for the nearest absolute maximum value to the local maximum value can obtain the MAX2, the determination of MAX2 is based on the difference between the maximum and the minimum according to step 2.

$$
\text { Period } \mathrm{T}=\left[\operatorname{MAX}_{[n+1]}-M A X_{[n]}\right]^{*} 0.01
$$

Step 4 The begin point of the minimum acceleration subscript is recorded. As the MIN1 in figure, look backward for the nearest absolute minimum value to the local minimum value can obtain the MIN2, the determination of MIN2 is based on the difference between the maximum and the minimum according to step 2.

$$
\text { Period } \mathrm{T}=\left[M \mathrm{IN}_{[n+1]}-M \mathrm{IN}_{[n]}\right] * 0.01
$$

\section{Gravity acceleration calibration}

The acquired acceleration signal value is the sum of the longitudinal acceleration and the gravity acceleration (or longitudinal component) of the sucker rod, it can be represented as:

$$
\mathrm{A}(t)=a(t)+g
$$

Where, A (t) is the acceleration value, a (t) is the longitudinal acceleration of the sucker rod, and $g$ is the gravity acceleration.

Due to differences in sensors and deviations in installation, there are differences of $g$ in the different oil wells ${ }^{[5]}$. This difference will be magnified after second order integral, thus affecting the final accuracy. Therefore, the acceleration must be zero point corrected. The static storage $\mathrm{g}$ has been tested, but due to the use of static $\mathrm{g}$ zero-point correction will still result in residual, and can not be changed according to different pumping units, so that the final calculation of the displacement has a greater error. According to the movement characteristics of the pumping unit, the method of dynamic zero-point correction is proposed.

This method can dynamically adjust the calculated gravity acceleration according to the types of pumping 
units. v ( $\mathrm{t}$ ) is the speed of the sucker rod, the time is $t$, and the integral of a $(\mathrm{t})$ in one period is

$$
\mathrm{v}(\mathrm{T})=\mathrm{v}(0)+\int_{0}^{\mathrm{T}} a(\tau) d \tau
$$

From the formula (14), (15), we can get

$$
\mathrm{v}(\mathrm{T})=\mathrm{v}(0)+\int_{0}^{\mathrm{T}}[A(\tau)-g] d \tau
$$

From the formula (10), (16), we can get

$$
\mathrm{g}=\frac{1}{\mathrm{~T}} \int_{0}^{\mathrm{T}} \mathrm{A}(\tau) d \tau
$$

The gravity acceleration $\mathrm{g}$ of an oil pumping unit is the average value of a periodic acceleration signal.

\section{The displacement is obtained by discrete numerical integration}

According to the determined period, the acceleration signal is discretized by numerical integration to obtain displacement ${ }^{[6]}$. The speed is calculated by integrating the acceleration of a complete period at one time, and the displacement is obtained by integrating the speed. There are many methods of discrete numerical integration, the Simpson formula is used in second order integral to obtain displacement as shown in the following formula ${ }^{[7]}$.

$$
\int_{a}^{b} f(x) d x \approx \int_{a}^{b} P_{2}(x) d x=\frac{b-a}{6}\left[f(a)+4 f\left(\frac{a+b}{2}\right)+f(b)\right]
$$

The speed is obtained by first order integrating the acceleration through Simpson formula according to formula (18), the speed after further integration is obtained by zero points correct to it, then integrating the speed once again.

$$
\mathrm{v}(\mathrm{t})=\mathrm{v}(0)-\frac{1}{\mathrm{~T}} \int_{0}^{\mathrm{T}} v(\tau) d \tau
$$

$\mathrm{V}(0)$ is the initial value of the speed, the beginning point of the relative displacement is zero, the relative displacement can be obtained by integrating V (T) with Simpson formula:

$$
\mathrm{d}(t)=\int_{0}^{t} v(\tau) d \tau
$$

Figure 5 is the displacement graph calculated according to the above formula, the practice measured displacement is $3.3 \mathrm{~m}$. The displacement calculated from the acceleration is $3.28 \mathrm{~m}$. In order to increase the persuasiveness, a series of experimental measurements have been carried out by this method, the data are shown in Table 1.

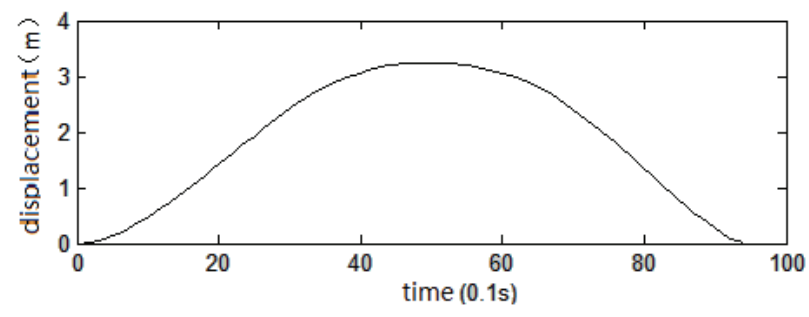

Figure 5. The Periodic displacement diagram.
Table 1. The displacement of Kalman filter measurement analysis

(displacement unit is $\mathrm{m}$; Scale measurement $3.3 \mathrm{~m}$ )

\begin{tabular}{|c|c|c|}
\hline $\begin{array}{c}\text { Measured } \\
\text { times }\end{array}$ & $\begin{array}{c}\text { The measured } \\
\text { displacement }\end{array}$ & $\begin{array}{c}\text { The relative } \\
\text { error }\end{array}$ \\
\hline 1 & 3.28 & $0.60 \%$ \\
\hline 2 & 3.29 & $0.30 \%$ \\
\hline 3 & 3.31 & $0.30 \%$ \\
\hline 4 & 3.33 & $0.90 \%$ \\
\hline 5 & 3.28 & $0.60 \%$ \\
\hline 6 & 3.32 & $0.60 \%$ \\
\hline 7 & 3.31 & $0.30 \%$ \\
\hline 8 & 3.27 & $0.90 \%$ \\
\hline
\end{tabular}

Table 2. The displacement of 7-point moving filtering measurement analysis

(displacement unit is $\mathrm{m}$; Scale measurement $3.3 \mathrm{~m}$ )

\begin{tabular}{|c|c|c|}
\hline $\begin{array}{c}\text { Measured } \\
\text { times }\end{array}$ & $\begin{array}{c}\text { The measured } \\
\text { displacement }\end{array}$ & $\begin{array}{c}\text { The relative } \\
\text { error }\end{array}$ \\
\hline 1 & 3.34 & $1.20 \%$ \\
\hline 2 & 3.199 & $3.30 \%$ \\
\hline 3 & 3.31 & $0.30 \%$ \\
\hline 4 & 3.43 & $33.90 \%$ \\
\hline 5 & 3.38 & $2.40 \%$ \\
\hline 6 & 3.42 & $3.60 \%$ \\
\hline 7 & 3.32 & $0.60 \%$ \\
\hline 8 & 3.24 & $1.80 \%$ \\
\hline
\end{tabular}

\section{CONCLUSION}

Compared with the most commonly used 7-point moving filtering, the field experiment results show that the Kalman filtering algorithm owns smaller maximum error and higher repeatability, which satisfies the requirement of relevant accuracy of oil well dynamometer card.

The displacement period searching method which proposed in this paper provides a reliable and basis method for the calculation of displacement, and improves the accuracy of displacement calculation. The zero point correction to the dynamic acceleration sensor data acquisition not only eliminates the residual of gravity acceleration, but also can be dynamically adjusted according to the different pumping unit and installation environment, this method is more suitable for different types of oil pumping unit. The displacements computed by the discrete numerical integral through the Simpson formula have smaller relative errors and higher accuracy.

\section{Acknowledgements}

This research is supported by National Natural Science Foundation of China under Grants 61501005, the Program of Educational Commission of Anhui Province under Grants KJ2017A104 and Science and Technology Projects of Production under Grant No.2015cxy03, which are gratefully acknowledged. 


\section{References}

1. PENG Jian, XU Zhi qiang, ZHOU Zhi quan. Design of Dynamometer Based on Sensor Technology[J]. Chinese Journal of Sensors and Actuators,2012,25(12):1701-1705.

2. WU Xin-sheng, TANG Ping, QIN Han. The application of digital filtering technique in signal acquisition of weighing system[J].Transducer and Microsystem Technologies,2010,29(09):131-134

3. Han Ping, Sang Wei lin, Shi Qing yan. Novel nonlinear Kalman filtering method[J]. Chinese Journal of Scientific Instrument,2015,36(03):632638

4. Jian min Duan, Hui Shi, Dan Liu, Hong xiao Yu. Square Root Cubature Kalman Filter-Kalman Filter Algorithm for Intelligent Vehicle Position Estimate[J]. Procedia Engineering,2016, 137:.

5. Kailei Liu, Zhi jia Li, Cheng Yao, Ji Chen, Ke Zhang, Muhammad Saifullah. Coupling the $\mathrm{k}$-nearest neighbour procedure with the Kalman filter for realtime updating of the hydraulic model in flood forecasting[J]. International Journal of Sediment Research, 2016,:

6. Peter Matisko, Vladimír Havlena. Optimality tests and adaptive Kalman filter[J]. IFAC Proceedings Volumes,2012,45(16):

7. Simo Särkkä,Arno Solin. On Continuous-Discrete Cubature Kalman Filtering[J]. IFAC Proceedings Volumes,2012,45(16): 